\title{
Commutation relations for functions of operators
}

Mark K. Transtrum

mktranstrum@byu.edu

Jean-Francois S. Van Huele

Follow this and additional works at: https://scholarsarchive.byu.edu/facpub

Part of the Astrophysics and Astronomy Commons, and the Physics Commons

\section{Original Publication Citation}

Transtrum, Mark K. and Jean."Commutation relations for functions of operators." Journal of Mathematical Physics 46 (25).

\section{BYU ScholarsArchive Citation}

Transtrum, Mark K. and Van Huele, Jean-Francois S., "Commutation relations for functions of operators" (2005). Faculty Publications. 372.

https://scholarsarchive.byu.edu/facpub/372

This Peer-Reviewed Article is brought to you for free and open access by BYU ScholarsArchive. It has been accepted for inclusion in Faculty Publications by an authorized administrator of BYU ScholarsArchive. For more information, please contact ellen_amatangelo@byu.edu. 


\title{
Commutation relations for functions of operators
}

\author{
Mark K. Transtrum ${ }^{\text {a) }}$ and Jean-François S. Van Huele ${ }^{\text {b) }}$ \\ Department of Physics and Astronomy, Brigham Young University, Provo, Utah 84602
}

(Received 18 January 2005; accepted 4 April 2005; published online 2 June 2005)

We derive an expression for the commutator of functions of operators with constant commutations relations in terms of the partial derivatives of these functions. This result extends the well-known commutation relation between one operator and a function of another operator. We discuss the range of applicability of the formula with examples in quantum mechanics. (C) 2005 American Institute of Physics. [DOI: 10.1063/1.1924703]

\section{INTRODUCTION}

A characteristic feature of quantum theory is the appearance of noncommuting operators. It is perhaps most conspicuous in one-dimensional quantum mechanics where the position and momentum operators obey the canonical commutation relation

$$
[x, p] \equiv x p-p x=i \hbar .
$$

In the coordinate representation of wave mechanics where the position operator $x$ is realized by $x$ multiplication and the momentum operator $p$ by $\hbar / i$ times the derivation with respect to $x$, one can easily check that the canonical commutation relation Eq. (1) is identically satisfied by applying the commutation operator on a test wave function $\psi(x)$,

$$
[x, p] \psi(x)=x \frac{\hbar}{i} \frac{\mathrm{d} \psi(x)}{\mathrm{d} x}-\frac{\hbar}{i} \frac{\mathrm{d}}{\mathrm{d} x}(x \psi(x))=\frac{\hbar}{i} x \psi^{\prime}(x)-\frac{\hbar}{i} \psi(x)-\frac{\hbar}{i} x \psi^{\prime}(x)=i \hbar \psi(x) .
$$

For quantum mechanics in three-dimensional space the commutation relations are generalized to

$$
\left[x_{i}, p_{j}\right]=i \hbar \delta_{i, j}
$$

and augmented with new commutation relations

$$
\left[x_{i}, x_{j}\right]=\left[p_{i}, p_{j}\right]=0,
$$

expressing the independence of the coordinates and of the momenta in the different dimensions.

When independent quantum mechanical systems are combined to form larger systems such as multiparticle systems, the commutation rules for the operators within the individual systems are preserved and augmented with vanishing commutation relations for operators acting on the different systems. Tensor products of the quantum mechanical spaces and of the operators that operate on them accommodate this extension naturally.

In the Heisenberg picture of quantum mechanics where the operators carry the time dependence of the system under consideration, the evolution of an individual operator $A$ is determined by its commutator with the Hamiltonian operator $H$, the generator of the time evolution:

\footnotetext{
${ }^{a)}$ Electronic mail: mktranstrum@hotmail.com

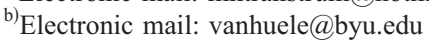




$$
\frac{d A}{d t}=\frac{1}{i \hbar}[A, H]=0 .
$$

Depending on the specific expression of the operators $A$ and $H$ in terms of the position and momentum operators, the evaluation of the commutators in a set of equations like Eq. (5) can become quite involved with implications on the integrability of the resulting differential equations.

The importance of evaluating commutators in quantum mechanics and the corresponding problem in quantum field theory is illustrated by the vast literature on the subject. Many sources and textbooks in quantum mechanics, starting with Dirac's seminal text [Dirac (1958)], state the canonical commutation relations either by postulating them, or by deriving them from their classical analogs, the canonical Poisson brackets, and then go on to show that they imply the following commutation between the position operator $x$ and any reasonable function of the momentum operator $f(p)$ :

$$
[x, f(p)]=i \hbar f^{\prime}(p)
$$

and its symplectic twin

$$
[p, f(x)]=-i \hbar f^{\prime}(x),
$$

where the (') symbol denotes differentiation with respect to the variable. The derivation of Eqs. (6) and (7) is a typical and almost obligatory exercise in a modern text on quantum mechanics. The standard way of proceeding is to consider the commutator of $x$ with increasing powers of $p$, to use induction, and to develop a Taylor expansion of the function $f$.

It may come as a surprise therefore that a further generalization of Eqs. (6) and (7) is never given. In fact we were not able to find an expression for the commutator of a function of $x$ with a function of $p$ in the literature. The present paper provides such an expression [Eq. (18) below when applied to $x_{1}=x$ and $\left.x_{2}=p\right]$ and goes a few steps further by giving the general commutator for functions of an arbitrary number of operators, with the restriction that all these operators have known commutation relations amongst themselves and that their commutators do not involve further operators (q-numbers) but only constants (c-numbers). To be specific, our formula does apply to functions of standard position and momentum operators in multiple dimensions and for multiple particles, as well as for functions of multiple raising and lowering (creation and annihilation) ladder operators, but it does not generally apply, for example, to functions of angular momentum operators. When dealing with angular momentum operators, one would need to reexpress them as functions of position and momentum, and then apply the formula to those operators directly. It does apply to functions of noncommuting position and momentum operators as considered in noncommutative space-time extensions of quantum theory [Snyder (1947), Jackiw (2001)] but only to the extent that the noncommutative operators obey constant, i.e., c-number, commutation relations.

In this paper, our approach to the problem is constructive. We state our general result first, consider simple cases, make use of the induction process, and proceed by generalization. Unless otherwise noted, we assume that the functions of the operators are well behaved in the sense that they can be expanded in Taylor series. In Sec. V we comment on the applicability of the formulas and give specific examples. In the Appendix we discuss correlations in the values of some summation indices that appear in our formulas and on the notation we developed to accommodate them.

\section{ASSUMPTIONS}

We begin by stating the definition of the commutator and list two properties that follow directly from this definition. The definition of the commutator, Eq. (8), linearity, Eq. (9), and Leibnitz's rule, Eq. (10), will all be used explicitly in our derivations

$$
[A, B]=A B-B A,
$$




$$
\begin{gathered}
{[A, \alpha B+C]=\alpha[A, B]+[A, C],} \\
{[A B, C]=A[B, C]+[A, C] B .}
\end{gathered}
$$

In addition to the above-noted relations, we will use a third, well-known commutator relation between an operator and a function of another operator. Given two operators $x_{1}$ and $x_{2}$ with a constant commutation relation $c$, such that $\left[x_{1}, x_{2}\right]=c$, it can be shown that

$$
\left[x_{1}, f\left(x_{2}\right)\right]=c f^{\prime}\left(x_{2}\right),
$$

where $f\left(x_{2}\right)$ is an analytic function with a Taylor expansion. We will accept this relation here and refer to our introductory section or to a text on quantum mechanics, e.g., Merzbacher (1998), for an outline of its derivation. For the canonical position and momentum in quantum mechanics, the constant $c$ takes the value $i \hbar$.

\section{GENERAL RESULT}

The most general result that we present in this paper involves the commutator of two functions of an arbitrary number of operators. Given $n$ operators $x_{1}, \ldots, x_{n}$ such that $\left[x_{i}, x_{j}\right]=c_{i j}$, where $c_{i j}$ are c-numbers, we will show that

$$
\begin{aligned}
{\left[f\left(x_{1}, \ldots, x_{n}\right), g\left(x_{1}, \ldots, x_{n}\right)\right]=} & \underbrace{}_{k_{1,2} \sum_{k_{1,3}} \sum \ldots k_{k_{2,3}} \ldots \sum_{k_{n-1, n}}\left(\prod_{j=2}^{n} \prod_{i=1}^{j-1} \frac{\left(-c_{i j}\right)^{k_{i j}}}{k_{i j} !}\right)} \\
& \times\left(\partial_{x_{1}}^{k_{1}} \ldots \partial_{x_{n}}^{k_{n}} g \partial_{x_{1}}^{k_{1}^{\prime}} \ldots \partial_{x_{n}}^{k_{n}^{\prime}} f-\partial_{x_{1}}^{k_{1}} \ldots \partial_{x_{n}}^{k_{n}} f \partial_{x_{1}}^{k_{1}^{\prime}} \ldots \partial_{x_{n}}^{k_{n}^{\prime}} g\right),
\end{aligned}
$$

where

$$
k_{i}=\sum_{j=i+1}^{n} k_{i j}
$$

and

$$
k_{i}^{\prime}=\sum_{j=1}^{i-1} k_{j i}
$$

The index of each summation ranges from zero to infinity $(i=0-\infty)$ with the restriction that the indices within the underbrace $(\sim)$ are not all simultaneously zero. We will use the underbrace throughout the paper to indicate this restriction. We refer to the Appendix for a further discussion of the summation indices and the underbrace. We notice that only dummy indices $k_{i j}$ with $i<j$ appear in the summation.

If the restriction that the indices cannot be simultaneously zero did not apply, there would be a term with no partial derivatives on the right-hand side of Eq. (12). That term would be $g f-f g$ $=[g, f]=-[f, g]$. Therefore, an equivalent statement of Eq. (12) reads

$$
\sum_{k_{1,2}=0}^{\infty} \sum_{k_{1,3}=0}^{\infty} \sum_{k_{2,3}=0}^{\infty} \ldots \sum_{k_{n-1, n}=0}^{\infty}\left(\prod_{j=2}^{n} \prod_{i=1}^{j-1} \frac{\left(-c_{i j}\right)^{k_{i j}}}{k_{i j} !}\right)\left(\partial_{x_{1}}^{k_{1}} \ldots \partial_{x_{n}}^{k_{n}} g \partial_{x_{1}}^{k_{1}^{\prime}} \ldots \partial_{x_{n}}^{k_{n}^{\prime}} f-\partial_{x_{1}}^{k_{1}} \ldots \partial_{x_{n}}^{k_{n}} f \partial_{x_{1}}^{k_{1}^{\prime}} \ldots \partial_{x_{n}}^{k_{n}^{\prime}} g\right)=0 .
$$

We will derive the generalized commutation relation Eq. (12), and we note that Eq. (15) is an equivalent, more compact statement that does not contain commutators of functions explicitly. 
All the results presented in this paper are special cases of this general result. We derive the result by constructing increasingly complex intermediate results in Sec. IV.

\section{DERIVATIONS}

We start by looking at the effect of increasing the power of $x_{1}$ in Eq. (11),

$$
\begin{aligned}
{\left[x_{1}^{2}, f\left(x_{2}\right)\right] } & =\left[x_{1}, f\left(x_{2}\right)\right] x_{1}+x_{1}\left[x_{1}, f\left(x_{2}\right)\right] \\
& =c f^{\prime}\left(x_{2}\right) x_{1}+x_{1} c f^{\prime}\left(x_{2}\right)=2 c x_{1} f^{\prime}\left(x_{2}\right)-c\left[x_{1}, f^{\prime}\left(x_{2}\right)\right]=2 c x_{1} f^{\prime}\left(x_{2}\right)-c^{2} f^{\prime \prime}\left(x_{2}\right) .
\end{aligned}
$$

We next show by induction that this result can be extended to an arbitrary power $n$,

$$
\left[x_{1}^{n}, f\left(x_{2}\right)\right]=\sum_{k=1}^{n}(-1)^{k+1}\left(\begin{array}{l}
n \\
k
\end{array}\right) c^{k} x_{1}^{n-k} f^{(k)}\left(x_{2}\right) .
$$

Proof of Eq. (17): Equations (11) and (16) correspond to the cases $n=1$ and $n=2$ of the result to be derived. We consider a general $n$ and expand the commutator using Leibnitz's rule applied to $x_{1}^{n}=x_{1} x_{1}^{n-1}$ :

$$
\begin{aligned}
{\left[x_{1}^{n}, f\left(x_{2}\right)\right]=} & x_{1}\left[x_{1}^{n-1}, f\left(x_{2}\right)\right]+\left[x_{1}, f\left(x_{2}\right)\right] x_{1}^{n-1} \\
= & \sum_{k=1}^{n-1}(-1)^{k+1}\left(\begin{array}{c}
n-1 \\
k
\end{array}\right) c^{k} x_{1}^{n-k} f^{(k)}\left(x_{2}\right)+c f^{\prime}\left(x_{2}\right) x_{1}^{n-1}=\sum_{k=1}^{n-1}(-1)^{k+1}\left(\begin{array}{c}
n-1 \\
k
\end{array}\right) c^{k} x_{1}^{n-k} f^{(k)}\left(x_{2}\right) \\
& +c x_{1}^{n-1} f^{\prime}\left(x_{2}\right)-\sum_{k=1}^{n-1}(-1)^{k+1}\left(\begin{array}{c}
n-1 \\
k
\end{array}\right) c^{k+1} x_{1}^{n-1-k} f^{(k+1)}\left(x_{2}\right)=n c x_{1}^{n-1} f^{\prime}\left(x_{2}\right) \\
& +\sum_{k=2}^{n-1}(-1)^{k+1}\left(\begin{array}{c}
n-1 \\
k
\end{array}\right) c^{k} x_{1}^{n-k} f^{(k)}\left(x_{2}\right)+\sum_{k=2}^{n-1}(-1)^{k+1}\left(\begin{array}{c}
n-1 \\
k-1
\end{array}\right) c^{k} x_{1}^{n-k} f^{(k)}\left(x_{2}\right) \\
& +(-1)^{n+1} c^{n} f^{(n)}\left(x_{2}\right)=\sum_{k=1}^{n}(-1)^{k+1}\left(\begin{array}{l}
n \\
k
\end{array}\right) c^{k} x_{1}^{n-k} f^{(k)}\left(x_{2}\right) .
\end{aligned}
$$

In the third line we used the identity $f^{\prime}\left(x_{2}\right) x_{1}^{n-1}=x_{1}^{n-1} f^{\prime}\left(x_{2}\right)-\left[x_{1}^{n-1}, f^{\prime}\left(x_{2}\right)\right]$, and in the fourth line we have redefined the index of the second summation to range from two to $n-1$ and have written the $n$th term outside the summation. In the final line we have combined all terms under a single summation. This completes the proof of Eq. (17).

We now extend the left argument of the commutator to include an analytic function of $x_{1}$ and proceed by considering its Taylor expansion

$$
\begin{aligned}
{\left[f\left(x_{1}\right), g\left(x_{2}\right)\right] } & =\left[\sum_{n=0}^{\infty} \frac{1}{n !} f^{(n)}(0) x_{1}^{n}, g\left(x_{2}\right)\right] \\
& =\sum_{n=0}^{\infty} \frac{1}{n !} f^{(n)}(0)\left[x_{1}^{n}, g\left(x_{2}\right)\right]=\sum_{n=0}^{\infty} \frac{1}{n !} f^{(n)}(0) \sum_{k=1}^{n}\left(\begin{array}{l}
n \\
k
\end{array}\right)(-1)^{(k+1)} c^{k} x_{1}^{n-k} g^{(k)}\left(x_{2}\right) .
\end{aligned}
$$

Interchanging the order of the summations, we find that we can write the result in terms of derivatives of the original function, $f\left(x_{1}\right)$. The result is

$$
\left[f\left(x_{1}\right), g\left(x_{2}\right)\right]=-\sum_{k=1}^{\infty} \frac{(-c)^{k}}{k !} f^{(k)}\left(x_{1}\right) g^{(k)}\left(x_{2}\right) .
$$

We pause to comment on this new result. The double summation introduced by the Taylor expansions has been reduced to a single sum. Somehow in the expression for the commutator of 
the two functions only the contributions from products of identical orders of derivatives survive. In a quantum mechanical context we see that the powers of position and momentum decrease simultaneously and make room for factors of $i \hbar$ factors as required by dimensional analysis.

We will now consider two analytic functions of two operators, $f\left(x_{1}, x_{2}\right)$ and $g\left(x_{1}, x_{2}\right)$. We want to write these as some expansion. We could use the one-dimensional Taylor expansion in $x_{1}$ and write

$$
f\left(x_{1}, x_{2}\right)=\sum_{n=0}^{\infty} \phi_{n}\left(x_{2}\right) x_{1}^{n},
$$

where the coefficients are now functions of the operator $x_{2}$. Instead, we simply observe that expansions exist (either as Taylor series or otherwise) and write them generally as

$$
f\left(x_{1}, x_{2}\right)=\sum_{n=0}^{\infty} \phi_{n}\left(x_{2}\right) f_{n}\left(x_{1}\right)
$$

and

$$
g\left(x_{1}, x_{2}\right)=\sum_{m=0}^{\infty} \gamma_{m}\left(x_{2}\right) g_{m}\left(x_{1}\right) .
$$

Since $x_{1}$ and $x_{2}$ do not commute we have made a choice in Eqs. (19) and (20) in writing all the $x_{2}$-dependence to the left of the $x_{1}$-dependence in every term in the sum. The ordering does affect the explicit expression for $\phi_{n}$ and $\gamma_{m}$ but the final result can be obtained no matter what ordering is chosen. What matters is that one choice has been made and will be used consistently throughout the derivation. The final result will be formally independent of the specific choice of $\phi_{n}, f_{n}, \gamma_{n}$, and $g_{n}$ and will be expressed in terms of $f\left(x_{1}, x_{2}\right)$ and $g\left(x_{1}, x_{2}\right)$ only.

Now we can consider the commutator:

$$
\begin{aligned}
{\left[f\left(x_{1}, x_{2}\right), g\left(x_{1}, x_{2}\right)\right]=} & \sum_{n=0}^{\infty} \sum_{m=0}^{\infty}\left[\phi_{n}\left(x_{2}\right) f_{n}\left(x_{1}\right), \gamma_{m}\left(x_{2}\right) g_{m}\left(x_{1}\right)\right] \\
= & \sum_{n=0}^{\infty} \sum_{m=0}^{\infty}\left(\phi_{n}\left(x_{2}\right) \gamma_{m}\left(x_{2}\right)\left[f_{n}\left(x_{1}\right), g_{m}\left(x_{1}\right)\right]+\phi_{n}\left(x_{2}\right)\left[f_{n}\left(x_{1}\right), \gamma_{m}\left(x_{2}\right)\right] g_{m}\left(x_{1}\right)\right. \\
& \left.+\left[\phi_{n}\left(x_{2}\right), \gamma_{m}\left(x_{2}\right)\right] g_{m}\left(x_{1}\right) f_{n}\left(x_{1}\right)+\gamma_{m}\left(x_{2}\right)\left[\phi_{n}\left(x_{2}\right), g_{m}\left(x_{1}\right)\right] f_{n}\left(x_{1}\right)\right)
\end{aligned}
$$

and notice that simplification occurs because the commutators of two functions of the same operator vanish, resulting in

$$
\left[f\left(x_{1}, x_{2}\right), g\left(x_{1}, x_{2}\right)\right]=\sum_{n=0}^{\infty} \sum_{m=0}^{\infty}\left(\phi_{n}\left(x_{2}\right)\left[f_{n}\left(x_{1}\right), \gamma_{m}\left(x_{2}\right)\right] g_{m}\left(x_{1}\right)+\gamma_{m}\left(x_{2}\right)\left[\phi_{n}\left(x_{2}\right), g_{m}\left(x_{1}\right)\right] f_{n}\left(x_{1}\right)\right) \text {. }
$$

Now we are in a position to apply Eq. (18) for two functions of a single operator each

$$
\begin{aligned}
{\left[f\left(x_{1}, x_{2}\right), g\left(x_{1}, x_{2}\right)\right]=} & \sum_{n=0}^{\infty} \sum_{m=0}^{\infty}\left(\phi_{n}\left(x_{2}\right)\left(-\sum_{k=1}^{\infty} \frac{(-c)^{k}}{k !} f_{n}^{(k)}\left(x_{1}\right) \gamma_{m}^{(k)}\left(x_{2}\right)\right) g_{m}\left(x_{1}\right)\right. \\
& \left.+\gamma_{m}\left(x_{2}\right)\left(\sum_{k=1}^{\infty} \frac{(-c)^{k}}{k !} g_{m}^{(k)}\left(x_{1}\right) \phi_{n}^{(k)}\left(x_{2}\right)\right) f_{n}\left(x_{1}\right)\right)
\end{aligned}
$$

Interchanging the order of the summations and factoring without commuting, we obtain 


$$
\begin{aligned}
{\left[f\left(x_{1}, x_{2}\right), g\left(x_{1}, x_{2}\right)\right]=} & -\sum_{k=1}^{\infty}\left(\frac{(-c)^{k}}{k !} \sum_{n=0}^{\infty} \phi_{n}\left(x_{2}\right) f_{n}^{(k)}\left(x_{1}\right) \sum_{m=0}^{\infty} \gamma_{m}^{(k)}\left(x_{2}\right) g_{m}\left(x_{1}\right)\right) \\
& +\sum_{k=1}^{\infty}\left(\frac{(-c)^{k}}{k !} \sum_{m=0}^{\infty} \gamma_{m}\left(x_{2}\right) g_{m}^{(k)}\left(x_{1}\right) \sum_{n=0}^{\infty} \phi_{n}^{(k)}\left(x_{2}\right) f_{n}\left(x_{1}\right)\right) .
\end{aligned}
$$

We now observe that the original function can be reassembled

$$
\sum_{n=0}^{\infty} \phi_{n}\left(x_{2}\right) f_{n}^{(k)}\left(x_{1}\right)=\frac{\partial^{k}}{\partial x_{1}^{k}}\left(\sum_{n=0}^{\infty} \phi_{n}\left(x_{2}\right) f_{n}\left(x_{1}\right)\right)=\frac{\partial^{k}}{\partial x_{1}^{k}} f\left(x_{1}, x_{2}\right)
$$

with similar formulas for partial derivatives of $f$ with respect to $x_{2}$, and for $g$ with respect to $x_{1}$ and $x_{2}$ and conclude that

$$
\left[f\left(x_{1}, x_{2}\right), g\left(x_{1}, x_{2}\right)\right]=\sum_{k=1}^{\infty} \frac{(-c)^{k}}{k !}\left(\frac{\partial^{k} g}{\partial x_{1}^{k}} \frac{\partial^{k} f}{\partial x_{2}^{k}}-\frac{\partial^{k} f}{\partial x_{1}^{k}} \frac{\partial^{k} g}{\partial x_{2}^{k}}\right) .
$$

In Eq. (22) we have omitted the arguments of the functions from the expression for brevity. We will continue to do so wherever there is no ambiguity in the arguments of the functions.

Before we can derive our formula for two functions of an arbitrary number of operators, we must prove one more formula giving the commutator of a function of an arbitrary number of operators and a function of one operator.

Given $n-1$ operators $x_{i}, i=1 \ldots(n-1)$ that each have a constant commutation relation with another given operator $x_{n}$, such that $\left[x_{i}, x_{n}\right]=c_{i n}$ then

$$
\left[f\left(x_{1}, \ldots, x_{n-1}\right), g\left(x_{n}\right)\right]=-\underbrace{\sum_{k_{1}} \cdots \sum_{k_{n-1}}}\left(\prod_{i=1}^{n-1} \frac{\left(-c_{i n}\right)^{k_{i}}}{k_{i} !}\right)\left(\partial_{x_{1}}^{k_{1}} \ldots d_{x_{n-1}}^{k_{n-1}} f d_{x_{n}}^{k} g\right),
$$

where

$$
k=\sum_{i=1}^{n-1} k_{i}
$$

We prove Eq. (23) by induction.

For $n=2 \mathrm{Eq}$. (23) becomes

$$
[f\left(x_{1} \ldots x_{n-1}, g\left(x_{n}\right)\right]=-\underbrace{\sum_{i=1}}_{k_{1}}\left(\prod_{i=1}^{1} \frac{\left(-c_{1,2}\right)^{k_{i}}}{k_{i} !}\right) \partial_{x_{\mathrm{r}}}^{k_{1}} f \partial_{x_{2}}^{k} g=-\sum_{k=1}^{\infty} \frac{\left(-c_{1,2}\right)^{k}}{k !} \partial_{x_{1}}^{k} f \partial_{x_{2}}^{k} g
$$

which we recognize as equivalent to Eq. (18), derived earlier.

Now we consider an arbitrary $n$ and, in analogy with Eq. (19), write

$$
f\left(x_{1}, \ldots, x_{n-1}\right)=\sum_{m=0}^{\infty} \phi_{m}\left(x_{n-1}\right) f_{m}\left(x_{1}, \ldots, x_{n-2}\right) .
$$

We expand the commutator using Leibnitz's rule to obtain

$$
[f, g]=\sum_{m=0}^{\infty}\left(\phi_{m}\left[f_{m}, g\right]+\left[\phi_{m}, g\right] f_{m}\right) .
$$


We evaluate the first commutator using Eq. (23) since the left argument is now a function of $n-2$ operators. We evaluate the second commutator using Eq. (18). The result is

$$
\begin{aligned}
& {[f, g]=-\sum_{m=0}^{\infty}\left(\phi_{m} \sum_{k_{1}} \cdots \sum_{k_{n-2}}\left(\prod_{i=1}^{n-2} \frac{\left(-c_{i n}\right)^{k_{i}}}{k_{i} !}\right) \partial_{x_{1}}^{k_{1}} \ldots \partial_{x_{n-2}}^{k_{n-2}} f_{m} \partial_{x_{n}}^{k} g\right)} \\
& -\sum_{m=0}^{\infty} \sum_{k_{n-1}=1}^{\infty} \frac{\left(-c_{n-1, n}\right)^{\kappa_{n-1}}}{k_{n-1} !}\left(\partial_{x_{n-1}}^{k_{n-1}} \phi_{m}\right)\left(\partial_{x_{n}}^{k_{n-1}} g\right) f_{m}
\end{aligned}
$$

where

$$
k=\sum_{i=1}^{n-1} k_{i}
$$

In the second term we apply the definition of the commutator Eq. (8) and Eq. (23) since again it involves a function of $n-2$ operators,

$$
\left(\partial_{x_{n}}^{k_{n-1}} g\right) f_{m}=f_{m}\left(\partial_{x_{n}}^{k_{n-1} g}\right)+\underbrace{\sum_{k_{1}} \cdots \sum_{k_{n-2}}}\left(\prod_{i=1}^{n-2} \frac{\left(-c_{i n}\right)^{k_{i}}}{k_{i} !}\right)\left(\partial_{x_{1}}^{k_{1}} \ldots \partial_{x_{n-2}}^{k_{n-2}} f_{m}\right)\left(\partial_{x_{n}}^{k} g\right)
$$

In the last term, we have used $k$ defined in Eq. (25). Substituting this into Eq. (24) we have

$$
\begin{aligned}
& {[f, g]=-\sum_{m=0}^{\infty}(\phi_{m} \underbrace{\sum_{k_{1}} \cdots \sum_{k_{n-2}}}\left(\prod_{i=1}^{n-2} \frac{\left(-c_{i n}\right)^{k_{i}}}{k_{i} !}\right)\left(\partial_{x_{1}}^{k_{1}} \ldots \partial_{x_{n-2}}^{k_{n-2}} f_{m}\right)\left(\partial_{x_{n}}^{k} g\right))-\sum_{m=0}^{\infty}\left(\sum_{k_{n-1}=1}^{\infty}\left(\frac{\left(-c_{n-1, n}\right)^{k_{n-1}}}{k_{n-1} !}\right)\right.}
\end{aligned}
$$

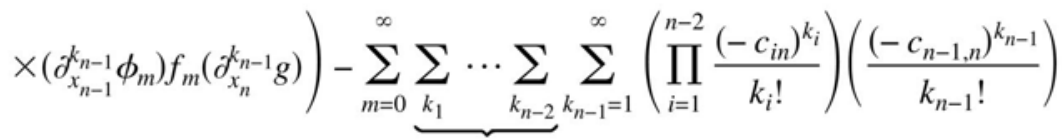

$$
\begin{aligned}
& \times\left(\partial_{x_{n-1}}^{k_{n-1}} \phi_{m}\right)\left(\partial_{x_{1}}^{k_{1}} \ldots \partial_{x_{n-2}}^{k_{n-2}} f_{m}\right)\left(\partial_{x_{n}}^{k} g\right) \text {. }
\end{aligned}
$$

Since all terms contain derivatives of $\phi_{m}$ next to derivatives of $f_{m}$, we can eliminate the summation over $m$ by using the observation made in Eq. (21) and write Eq. (27) in terms of the original function $f$. We also combine factors

$$
\left(\prod_{i=1}^{n-2} \frac{\left(-c_{i n}\right)^{k_{i}}}{k_{i} !}\right)\left(\frac{\left(-c_{n-1, n}\right)^{k_{n-1}}}{k_{n-1} !}\right)=\prod_{i=1}^{n-1} \frac{\left(-c_{i n}\right)^{k_{i}}}{k_{i} !}
$$

to get

$$
\begin{aligned}
& {[f, g]=-\underbrace{\sum_{k_{1}} \cdots \sum_{k_{n-2}}}\left(\prod_{i=1}^{n-2} \frac{\left(-c_{i n}\right)^{k_{i}}}{k_{i} !}\right)\left(\partial_{x_{1}}^{k_{1}} \ldots \partial_{x_{n-2}}^{k_{n-2}} f\right)\left(d_{x_{n}}^{k} g\right)-\sum_{k_{n-1}=1}^{\infty}\left(\frac{\left(-c_{n-1, n}\right)^{k_{n-1}}}{k_{n-1} !}\right)\left(d_{x_{n-1}}^{k_{n-1}} f\right)\left(\partial_{x_{n}}^{k_{n-1}} g\right)} \\
& -\underbrace{\sum_{k_{n-2}} \ldots \sum_{k_{n-1}=1}^{\infty}}_{k_{1}}\left(\prod_{i=1}^{n-1} \frac{\left(-c_{i n}\right)^{k_{i}}}{k_{i} !}\right)\left(\partial_{x_{1}}^{k_{1}} \ldots \partial_{x_{n-2}}^{k_{n-2}} \partial_{x_{n-\mathrm{r}}}^{k_{n-1}} f\right)\left(\partial_{x_{n}}^{k} g\right)
\end{aligned}
$$


We observe that in the first term, the indices of the summations are not simultaneously zero. In the last term, the first $n-2$ indices are not simultaneously zero. Thus, the first term corresponds to the case $k_{n-1}=0$. The second term is the case in which $k_{1} \ldots k_{n-2}$ are simultaneously zero, and the third term is the case in which the indices of neither group are simultaneously zero. Therefore, these three terms can be collected into one term as we elaborate in the Appendix. This concludes the proof of Eq. (23).

We are now ready to prove our main result, Eq. (12). This proof will again proceed by induction.

First, we consider the case $n=2$ :

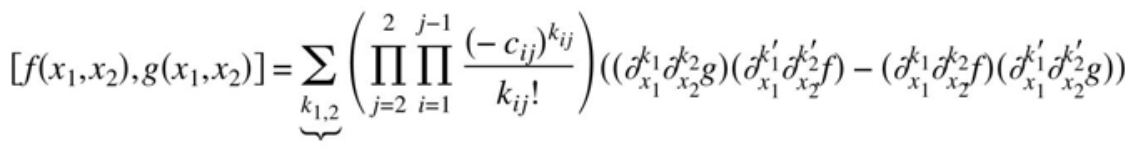

$$
\begin{aligned}
& =\sum_{k=1}^{\infty} \frac{\left(-c_{1,2}\right)^{k}}{k !}\left(\partial_{x_{1}}^{k} g \partial_{x_{2}}^{k} f-\partial_{x_{1}}^{k} f \partial_{x_{2}}^{k} g\right),
\end{aligned}
$$

where we have used Eqs. (13) and (14) to find that $k_{1}=k_{2}^{\prime}=k_{1,2}$ and $k_{2}=k_{1}^{\prime}=0$. We recognize that this is just the result derived in Eq. (22).

We now consider an arbitrary value of $n$. We write the functions in analogy with Eqs. (19) and

$$
\begin{aligned}
& f\left(x_{1}, \ldots, x_{n}\right)=\sum_{m=0}^{\infty} \phi_{m}\left(x_{n}\right) f_{m}\left(x_{1}, \ldots, x_{n-1}\right) \\
& g\left(x_{1}, \ldots, x_{n}\right)=\sum_{p=0}^{\infty} \gamma_{p}\left(x_{n}\right) g_{p}\left(x_{1}, \ldots, x_{n-1}\right) .
\end{aligned}
$$

Applying Leibnitz's rule to $[f, g]$ we have

$$
[f, g]=\sum_{m=0}^{\infty} \sum_{p=0}^{\infty}\left(\left[\phi_{m}, \gamma_{p}\right] g_{p} f_{m}+\gamma_{p}\left[\phi_{m}, g_{p}\right] f_{m}+\phi_{m}\left[f_{m}, \gamma_{p}\right] g_{p}+\phi_{m} \gamma_{p}\left[f_{m}, g_{p}\right]\right) .
$$

We observe that the first commutator vanishes since it involves the single operator $x_{n}$. We use Eq. (23) to evaluate the second and third terms. We use Eq. (12) to evaluate the last term, since both arguments are functions of $n-1$ operators. The result is

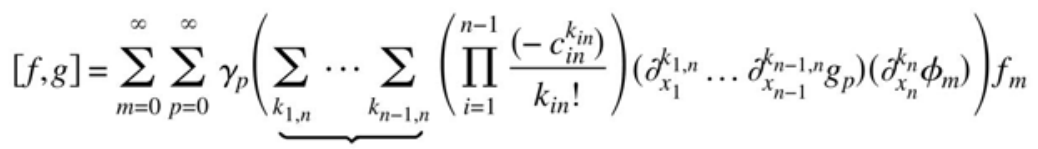

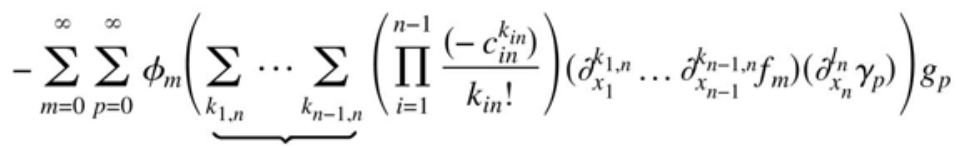

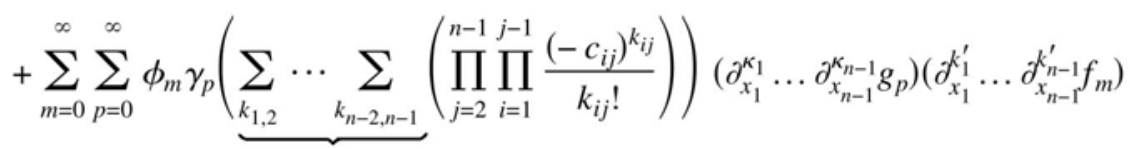

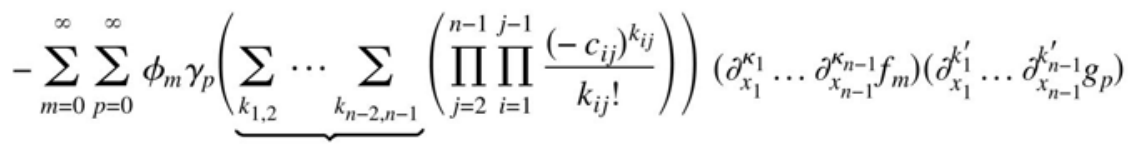

where we have used the abbreviation 


$$
\kappa_{i}=\sum_{j=i+1}^{n-1} k_{i j}
$$

and $k_{i}^{\prime}$ is defined in Eq. (14).

First, we notice that the first two terms of Eq. (29) can be combined:

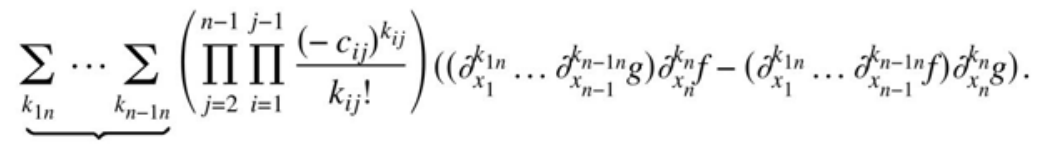

Next, we notice that $\phi_{m}$ and $\gamma_{p}$ commute. Also, using the definition of the commutator we can change the order of $\phi_{m}\left(\partial_{x_{1}}^{k_{1}} \ldots \partial_{x_{n-1}}^{k_{n-1}} g_{p}\right)$ and $\gamma_{p}\left(\partial_{x_{1}}^{k_{1}} \ldots \partial_{x_{n-1}}^{k_{n-1}} f_{m}\right)$. We use Eq. (23) to evaluate these commutators. After substitution into Eq. (29), the result is:

$$
\begin{aligned}
& {[f, g]=\underbrace{\sum_{k_{1 n}} \ldots \sum_{k_{n-1} n}}\left(\prod_{j=2}^{n-1} \prod_{i=1}^{j-1} \frac{\left(-c_{i j}\right)^{k_{i j}}}{k_{i j} !}\right)\left(\partial_{x_{1}}^{k_{1, n}} \ldots \partial_{x_{n-1}}^{k_{n-1, n}} g d_{x_{n}}^{k_{n}} f-d_{x_{1}}^{k_{1, n}} \ldots d_{x_{n-1}}^{k_{n-1, n}} f \partial_{x_{n}}^{k_{n} g}\right)}
\end{aligned}
$$

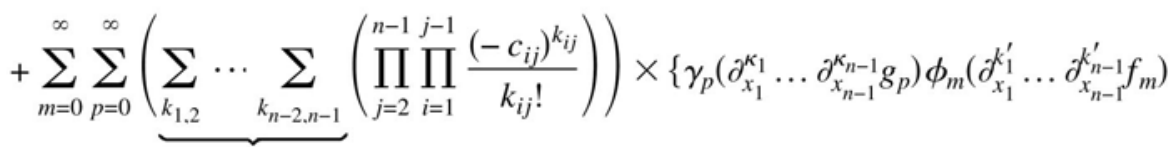

$$
\begin{aligned}
& \left.-\phi_{m}\left(\partial_{x_{1}}^{\kappa_{1}} \ldots \partial_{x_{n-1}}^{\kappa_{n-1}} f_{m}\right) \gamma_{p}\left(\partial_{x_{1}}^{k_{1}^{\prime}} \ldots \partial_{x_{n-1}}^{k_{n-1}^{\prime}} g_{p}\right)\right\}
\end{aligned}
$$

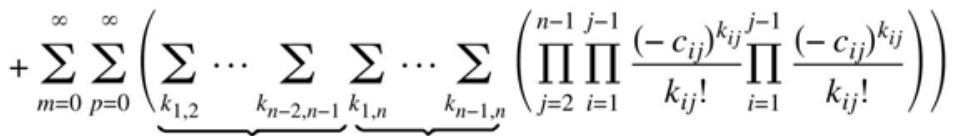

$$
\begin{aligned}
& \times\left\{\gamma_{p}\left(\partial_{x_{1}}^{\kappa_{1}+k_{1, n}} \ldots \partial_{x_{n-1} \kappa_{n-1}+k_{n-1, n}} g_{p}\right)\left(\partial_{x_{n}}^{k_{1, n}+\ldots+k_{n-1, n}} \phi_{m}\right)\left(\partial_{x_{1}}^{k_{1}^{\prime}} \ldots \partial_{x_{n-1}}^{k_{n-1}^{\prime}} f_{m}\right)\right. \\
& \left.-\phi_{m}\left(\partial_{x_{1}}^{\kappa_{1}+k_{1, n}} \ldots \partial_{x_{n-1}}^{\kappa_{n-1}+k_{n-1, n}} f_{m}\right)\left(\partial_{x_{n}}^{k_{1, n}+\ldots+k_{n-1, n}} \gamma_{p}\right)\left(\partial_{x_{1}}^{k_{1}^{\prime}} \ldots \partial_{x_{n-1}}^{k_{n-1}^{\prime} g_{p}}\right)\right\} \text {. }
\end{aligned}
$$

Combining factors

$$
\left(\prod_{j=2}^{n-1} \prod_{i=1}^{j-1} \frac{\left(-c_{i j}\right)^{k_{i} j}}{k_{i j} !}\right)\left(\prod_{i=1}^{n-1} \frac{\left(-c_{i n}\right)^{k_{i n}}}{k_{i n} !}\right)=\prod_{j=2}^{n} \prod_{i=1}^{j-1} \frac{\left(-c_{i j}\right)^{k_{i j}}}{k_{i j} !}
$$

and rearranging the summations, we get 


$$
\begin{aligned}
& {[f, g]=\underbrace{\sum_{k_{1, n}} \cdots \sum_{k_{n-1, n}}}\left(\prod_{j=2}^{n-1} \prod_{i=1}^{j-1} \frac{\left(-c_{i j}\right)^{k_{i j}}}{k_{i j} !}\right)\left(\left(\partial_{x_{1}}^{k_{1, n}} \ldots \partial_{x_{n-1}}^{k_{n-1, n} g} g\right)\left(d_{x_{n}}^{k_{n}} f\right)-\left(\partial_{x_{1}}^{k_{1, n}} \ldots \partial_{x_{n-1}}^{k_{n-1, n}} f\right)\left(d_{x_{n}}^{k_{n}} g\right)\right)}
\end{aligned}
$$

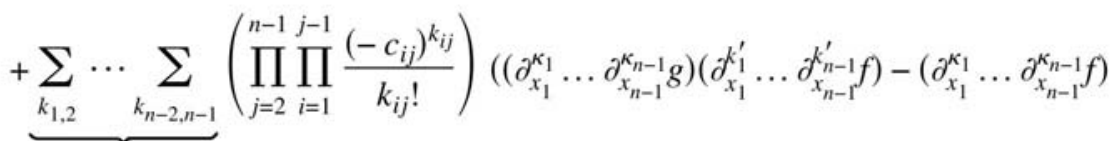

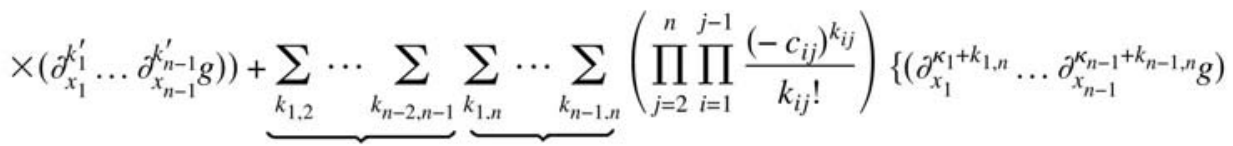

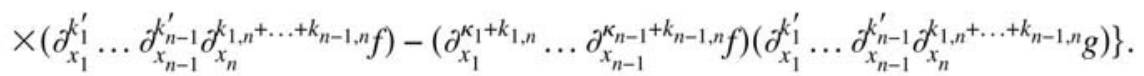

Now we observe that

$$
\kappa_{i}+k_{i n}=\sum_{j=i+1}^{n-1} k_{i j}+k_{i n}=k_{i}
$$

and that

$$
k_{1, n}+\ldots+k_{n-1, n}=k_{n}^{\prime}
$$

in accordance with Eqs. (13) and (14), which allows us to write

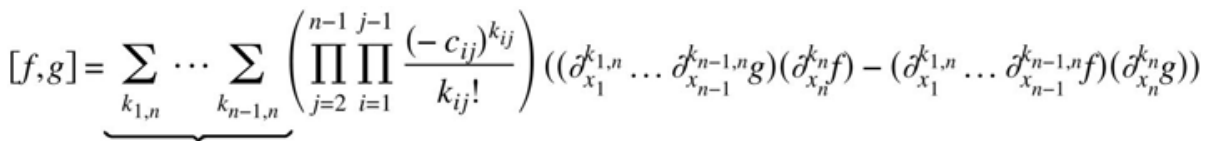

$$
\begin{aligned}
& +\underbrace{\sum_{k_{1,2}} \cdots \sum_{k_{n-2, n-1}}}\left(\prod_{j=2}^{n-1} \prod_{i=1}^{j-1} \frac{\left(-c_{i j}\right)^{k_{i j}}}{k_{i j} !}\right)\left(\left(\partial _ { x _ { 1 } } ^ { k _ { 1 } } \ldots \partial _ { x _ { n - 1 } } ^ { k _ { n - 1 } g ) } \left(\partial_{x_{1}}^{k_{1}^{\prime}} \ldots \partial_{x_{n-1}}^{\left.k_{n-1}^{\prime} f\right)-\left(\partial_{x_{1}}^{k_{1}} \ldots \partial_{x_{n-1}}^{k_{n-1}} f\right)}\right.\right.\right.
\end{aligned}
$$

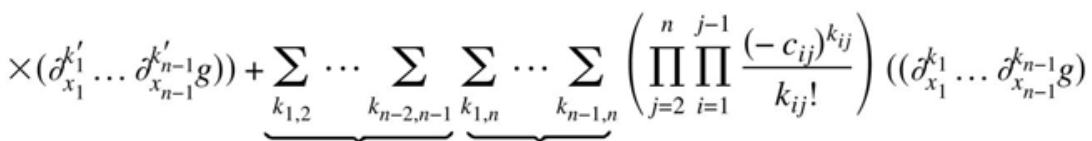

$$
\begin{aligned}
& \times\left(\partial_{x_{n}}^{k_{n}^{\prime}} \cdot k_{x_{1}}^{\prime} \ldots \partial_{x_{n-1}}^{\left.k_{n-1}^{\prime} f\right)}-\left(\partial_{x_{1}}^{k_{1}} \ldots \partial_{x_{n-1}}^{k_{n-1}} f\right)\left(d_{x_{n}}^{k_{n}} \partial_{x_{1}}^{\prime} \ldots \partial_{x_{n-1}}^{k_{n-1}^{\prime}} g\right)\right) \text {. }
\end{aligned}
$$

Finally, we observe the ranges of the groups of indices. The first term does not contain the indices $k_{1,2}, \ldots, k_{n-2, n-1}$ and, therefore, corresponds to the term in which they are simultaneously zero. Also, the indices $k_{1, n}, \ldots, k_{n-1, n}$ do not appear in the second term, which then corresponds to the term in which they are simultaneously zero. The third term has both sets of indices. Thus the three terms can be combined (see the Appendix) noting that all the indices range from zero to infinity, but are not simultaneously zero. Upon doing so, the equation becomes (12), completing the proof.

\section{DISCUSSION AND IMPLEMENTATION}

The main result of this paper is Eq. (12). It gives a formula for the commutator of two functions of noncommuting operators. It can actually be written more compactly in the form of Eq. (15) in which both the left-hand side and the constraints on the summation indices have been removed. Equation (15) actually looks so simple that one wonders if it cannot be derived trivially from some general statement, instead of through the long and convoluted induction processes 
presented in Sec. IV. We have not been able to do so. Equation (12) applies to reasonable functions $f$ and $g$ which have converging Taylor series. In the derivation, we interchanged the order of the summations, thereby replacing a series by another, a step that is valid when both series converge. We used the linearity of the derivative to derive term by term, a process that is again valid for converging series with converging derivatives. Of course all formulas apply also to all finite or truncated series such as those that appear when evaluating expressions to any finite order of perturbation theory for example. That is the spirit in which the formulas were derived and the area where they are most likely to find their domain of applicability. Since $x_{1}$ and $x_{2}$ in Eq. (22) and $x_{1}, x_{2} \ldots, x_{n}$ in Eq. (12) are operators, the derivatives and partial derivatives need to be explained further. Already in Eq. (11) the derivative symbol refers to a derivative with respect to an operator. In the one-dimensional case Eq. (11) can easily be interpreted as the operator replacement of the derivative of a function with respect to a scalar variable. The prescription becomes: take the ordinary derivative of the function and replace in the resulting expression every occurrence of the variable $x$ by an operator $X$. So for instance, denoting operators with capitals for now, the procedure to find the operator derivative in a particular case gives

$$
\left(e^{3 X}\right)^{\prime} \rightarrow\left(e^{3 x}\right)^{\prime}=3 e^{3 x} \rightarrow 3\left(e^{3 X}\right) .
$$

When the function depends on two or more noncommuting operators however, an operational definition of the derivative needs to be supplied. We follow Louisell (1964) to define the derivative with respect to an operator in terms of the derivative with respect to a scalar parameter $\lambda$ :

$$
\partial_{x} f(x, p)=\lim _{\lambda \rightarrow 0} \frac{\partial}{\partial \lambda} f(x+\lambda, p) .
$$

and

$$
\partial_{x_{i}} f\left(x_{1}, \ldots, x_{i}, \ldots, x_{n}\right)=\lim _{\lambda \rightarrow 0} \frac{\partial}{\partial \lambda} f\left(x_{1}, \ldots, x_{i}+\lambda, \ldots, x_{n}\right) .
$$

This definition reduces to the ordinary derivative shown above in the one-dimensional case:

$$
\partial_{x} e^{3 x}=\lim _{\lambda \rightarrow 0} \frac{\partial}{\partial \lambda} e^{3(x+\lambda)}=\lim _{\lambda \rightarrow 0} 3 e^{3(x+\lambda)}=3 e^{3 x} .
$$

Let us now apply this definition for operator derivatives to a function of two operators $x$ and $p$ as an example of a situation that might arise in the evaluation of Eq. (12). It is clear that, as a result of the noncommutativity of $x$ and $p$ the ordinary composition or chain rule,

$$
(f(g(x)))^{\prime}=f^{\prime}(g(x)) g^{\prime}(x)=g^{\prime}(x) f^{\prime}(g(x))
$$

cannot be applied without ambiguity since, for example,

$$
\partial_{x}(\cos (x p)) \neq-\sin (x p) p \neq p(-\sin (x p) \neq 1 / 2(-\sin (x p) p+p(-\sin (x p)) .
$$

For the exponential function however, we can follow Wilcox (1967) and adapt a result from Snider (1964) to obtain

$$
\partial_{x}\left(e^{f(x, p)}\right)=\int_{0}^{1} \mathrm{~d} u e^{(1-u) f(x, p)}\left(\partial_{x} f(x, p)\right) e^{u f(x, p)}
$$

which can itself be used to get an explicit expression for the derivative operator in Eq. (35) above

$$
\partial_{x}(\cos (x p))=-\int_{0}^{1} \mathrm{~d} u \cos [(1-u) x p] p \sin (u x p)-\int_{0}^{1} \mathrm{~d} u \sin [(1-u) x p] p \cos (u x p) .
$$


This result is indeed quite different from that obtained from a naive application of the chain rule for derivatives with respect to commuting variables. Furthermore the result in Eq. (37) can be checked to any order of an expansion in powers of $x$ or $p$. The derivative of a polynomial truncation of the cosine function yields the result obtained by truncation of the trigonometric functions in Eq. (37). We have developed algorithms for manipulating any polynomial expression of noncommuting operators [Transtrum and Van Huele (to be published)]. That linearity and Leibnitz's rule apply to the operational derivative follows from the fact that these properties are preserved in Eq. (31).

Finally we use Eq. (12) to derive the torque equation in the Heisenberg picture of quantum mechanics as an illustration of a commutator of two functions, one of which (only) can be expanded in a finite power series of its operators.

Consider the commutator of the nonrelativistic Hamiltonian,

$$
H=\frac{p_{x}^{2}+p_{y}^{2}+p_{z}^{2}}{2 m}+V(x, y, z),
$$

with the $z$ component of the angular momentum, $L_{z}=x p_{y}-y p_{x}$. Since the only nonvanishing commutators are $\left[x, p_{x}\right]=\left[y, p_{y}\right]=\left[z, p_{z}\right]=i \hbar$, the formula simplifies to

$$
\begin{aligned}
{\left[L_{z}\left(x, y, p_{x}, p_{y}\right), H\left(x, y, z, p_{x}, p_{y}, p_{z}\right)\right]=} & \underbrace{\sum_{k} \sum_{l} \sum_{m} \frac{(-i \hbar)^{k+l+m}}{k ! l ! m !}}_{k} \\
& \times\left(\left(\partial_{x}^{k} \partial_{y}^{l} \partial_{z}^{m} H\right)\left(\partial_{p_{x}}^{k} \partial_{p_{y}}^{l} \partial_{p_{z}}^{m} L_{z}\right)-\left(\partial_{x}^{k} \partial_{y}^{l} \partial_{z}^{m} L_{z}\right)\left(\partial_{p_{x}}^{k} \partial_{p_{y}}^{l} \partial_{p_{z}}^{m} H\right)\right)
\end{aligned}
$$

By inspection, it is obvious that all mixed partial derivatives of $L_{z}$ that occur in the above formula vanish. So, the above formula further simplifies to

$$
\begin{aligned}
{\left[L_{z}, H\right]=} & \sum_{k=1}^{\infty} \frac{(-i \hbar)^{k}}{k !}\left(\left(\partial_{x}^{k} H\right)\left(\partial_{p_{x}}^{k} L_{z}\right)-\left(\partial_{x}^{k} L_{z}\right)\left(\partial_{p_{x}}^{k} H\right)\right)+\sum_{k=1}^{\infty} \frac{(-i \hbar)^{k}}{k !}\left(\left(\partial_{y}^{k} H\right)\left(\partial_{p_{y}}^{k} L_{z}\right)-\left(\partial_{y}^{k} L_{z}\right)\left(\partial_{p_{y}}^{k} H\right)\right) \\
& +\sum_{k=1}^{\infty} \frac{(-i \hbar)^{k}}{k !}\left(\left(\partial_{z}^{k} H\right)\left(\partial_{p_{z}}^{k} L_{z}\right)-\left(\partial_{z}^{k} L_{z}\right)\left(\partial_{p_{z}}^{k} H\right)\right) \\
= & (-i \hbar)\left(\left(\partial_{x} V\right)(-y)-p_{y} \frac{p_{x}}{m}+\left(\partial_{y} V\right)(x)-\left(-p_{x}\right) \frac{p_{y}}{m}\right)=(-i \hbar)\left(\left(\partial_{y} V\right) x-\left(\partial_{x} V\right) y\right)
\end{aligned}
$$

Therefore

$$
\frac{\mathrm{d} L_{z}}{\mathrm{~d} t}=\frac{1}{i \hbar}\left[L_{z}, H\right]=x F_{y}-y F_{x} .
$$

We recognize the torque about the $z$ axis as expected.

In conclusion, we have presented an expression for the commutator of two functions of an arbitrary number of noncommuting operators whose commutators are constants. The formula is derived constructively using the induction process. The resulting expression involves partial derivatives of the functions with respect to the noncommuting operators. These partial derivatives can be evaluated directly in some simple cases or in general through series expansions and ordering algorithms to any order.

\section{ACKNOWLEDGMENT}

We acknowledge support from the Office for Research and Creative Activities (ORCA) at Brigham Young University. 


\section{APPENDIX: NOTATION FOR SUMMATION INDICES}

In this appendix we comment on the use of our notation for the summation indices as they appear in Eqs. (12)-(14). First of all $k_{1}=k_{n}^{\prime}=0$ for any $n$. We include these terms to see the symmetry in the formula.

Second, we note that when the summation indices have two subscripts, we separate the subscripts by commas when at least one of them involves a number. Thus we write $k_{1,2}, k_{1, i}$, and $k_{i-1, j}$, but $k_{i j}$ instead of $k_{i, j}$. Similarly, we write $c_{1,2}, c_{1, i}$, and $c_{i-1, j}$, but $c_{i j}$ instead of $c_{i, j}$. The simplification $c_{1,2}=c$ was made in the part of Sec. IV leading to Eq. (22).

Finally we discuss the correlation between the summation indices and the related underbrace notation in more depth. Throughout the paper, we frequently use notation of the form

$$
\underbrace{\sum_{k_{1}} \cdots \sum_{k_{n}}} f\left(k_{1}, \ldots, k_{n}\right),
$$

where the underbrace indicates that the summations range from zero to infinity but are not simultaneously zero. We will explain some of the properties associated with these expressions.

In the case that the underbrace includes only one index, that index cannot be zero, and the expression can be rewritten in more standard notation:

$$
\underbrace{\sum_{1}}_{k_{1}} f\left(k_{1}\right)=\sum_{k_{1}=1}^{\infty} f\left(k_{1}\right)
$$

Now, we consider the case that the underbrace contains two indices. Both cannot be zero simultaneously, but one can be zero as long as the other is nonzero. Thus, there are two summations which account for the cases in which one of the indices is zero. There is also the case that neither index is zero and a third term accounts for this:

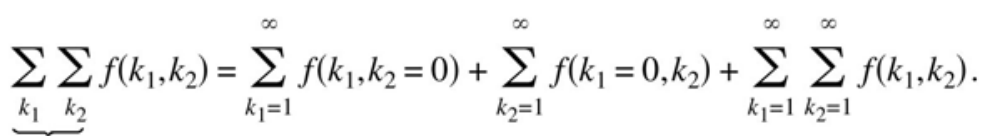

We observe here that this result implies that

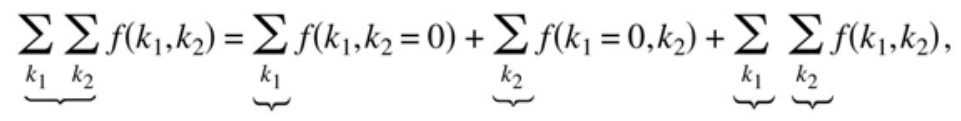

which we will generalize shortly.

It follows from the above argument, that, if there are $n$ indices, $k_{1}, \ldots, k_{n}$, in an expression of the form

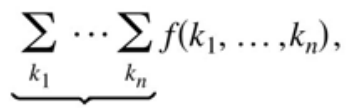

there will be $\left(\begin{array}{c}n \\ 1\end{array}\right)$ terms in which one index is nonzero, $\left(\begin{array}{c}n \\ 2\end{array}\right)$ terms in which two indices are nonzero, and so forth. Thus, there will be 


$$
\sum_{k=1}^{n}\left(\begin{array}{l}
n \\
k
\end{array}\right)=2^{n}-1
$$

terms altogether. For large numbers of indices, the underbrace notation is clearly more compact; however, for computational purposes, it will be necessary to expand the underbrace notation as we have done here for the cases $n=1$ and $n=2$.

Now we consider a situation that arises frequently in the derivations presented in this paper. This involves the case in which there are two groups of indices, $k_{1}, \ldots, k_{n}$ and $l_{1}, \ldots, l_{m}$ in an expression of the form

$$
\underbrace{}_{k_{k_{1}} \cdots \sum_{k_{n}} \sum_{l_{1}} \cdots \sum_{l_{m}} f}
$$

where $f$ depends on each of the indices. We show that the underbrace has the following property:

$$
\underbrace{\sum_{k_{1}} \cdots \sum_{k_{n}} \sum_{l_{1}} \cdots \sum_{l_{m}} f}_{k_{1}}=\underbrace{\sum_{k_{1}} \cdots \sum_{k_{n}} f}+\underbrace{\sum_{l_{1}} \cdots \sum_{l_{m}} f}+\underbrace{\sum_{k_{1}} \cdots \sum_{k_{n}}} \underbrace{\sum_{l_{1}} \cdots \sum_{l_{m}} f}
$$

which is an identity used often throughout the paper. Given a little thought, this expression seems reasonable, but we will present a slightly more rigorous proof.

The proof is not complicated; we simply count the number of terms on either side of the equation. The left-hand side of the equation has $2^{n+m}-1$ terms. The right-hand side has $2^{n}-1$ $+2^{m}-1+\left(2^{n}-1\right)\left(2^{m}-1\right)=2^{n+m}-1$ terms. Since all of the terms on the the right involve summations in which some of the indices range from zero to infinity but are not simultaneously zero, we conclude that each term on the right-hand side of the equation also appears on the left with no repeated terms. Since both sides of the equation have equal numbers of terms, we conclude that the two expressions are equivalent.

${ }^{1}$ Dirac, P. A. M., The Principles of Quantum Mechanics, 4th ed. revised (Oxford University Press, Oxford, 1958).

${ }^{2}$ Jackiw, R., "Physical instances of noncommuting coordinates," arXiv: hep-th/0111057 (2001).

${ }^{3}$ Louisell, W. H., Radiation and Noise in Quantum Electronics (McGraw-Hill, New York, 1964).

${ }^{4}$ Merzbacher, E., Quantum Mechanics, 3rd ed. (Wiley, New York, 1998).

${ }^{5}$ Snider, R. F., "Perturbation variation methods for a quantum Boltzmann equation," J. Math. Phys. 5, 1580-1587 (1964).

${ }^{6}$ Snyder, H. S., "Quantized space-time," Phys. Rev. 71, 38-41 (1947).

${ }^{7}$ Transtrum, M. K. and Van Huele, J.-F. S., "Algorithms for normal ordering polynomial functions of noncommuting operators and their Maple implementation," (unpublished).

${ }^{8}$ Wilcox, R. M., "Exponential operators and parameter differentiation in quantum physics," J. Math. Phys. 8, 962-982 (1964). 